\title{
Overproduction, Purification and Refolding of codon-optimized Hepatitis B Virus X Protein Subgenotype B3 in Escherichia coli BL21(DE3)
}

\author{
Anita Artarini ${ }^{1 *}$, Armini Syamsidi ${ }^{1}$, Anindyajati ${ }^{1}$, Raymond R. Tjandrawinata ${ }^{2}$, Debbie S. Retnoningrum $^{1 * *}$ \\ 'Laboratory of Pharmaceutical Biotechnology, School of Pharmacy, Institut Teknologi Bandung, Bandung, Indonesia \\ ${ }^{2}$ Dexa Laboratories of Biomolecular Sciences, Cikarang, Indonesia
}

\section{ARTICLE INFO}

Article history:

Received February 22, 2021

Received in revised form September 17, 2021

Accepted October 4, 2021

\section{KEYWORDS:}

HBV,

$\mathrm{Hbx}$,

Protein refolding,

Subgenotype B3,

Thermal shift assay

\begin{abstract}
Hepatitis B virus (HBV) infects human and causes chronic liver infection, leading to liver cirrhosis and hepatocellular carcinoma. HBV $\mathbf{X}(\mathrm{Hbx})$ protein is known to interact with tumor suppressor protein $\mathrm{p53}$ and block its translocation into the nucleus. This study outlines the overproduction of $\mathrm{Hbx}$ protein from $\mathrm{HBV}$ subgenotype $\mathrm{B3}$ in Escherichia coli BL21(DE3), including its purification and refolding. The gene encoding $\mathrm{Hbx}$ was first codon-optimized and inserted into pET16b. The recombinant plasmid was then transformed into $E$. coli BL21(DE3) as an expression host. Optimization of $\mathrm{Hbx}$ expression was performed with variation of IPTG concentration and overproduction temperature. The results showed that $\mathrm{Hbx}$ protein was optimally induced by $0.075 \mathrm{mM}$ IPTG and overproduction of $\mathrm{Hbx}$ at 17,25 , and $37^{\circ} \mathrm{C}$ exhibited no difference in protein level and location. The optimal refolding of $\mathrm{Hbx}$ was obtained using $0.1 \mathrm{M}$ arginine prior to elution from Nickel column using $100 \mathrm{mM}$ imidazole and $0.25 \mathrm{M}$ arginine. Hbx migrates differently in SDS-PAGE reducing and non-reducing, while the melting curve pattern in TSA analysis changed after the refolding step. Essentially, this purified Hbx protein could potentially be used for interaction study with p53 and the inhibitor candidate of the protein.
\end{abstract}

\section{Introduction}

Hepatitis B virus (HBV) belongs to the Hepadnaviridae family and is the causative agent of liver infection. HBV causes acute and chronic liver infection, where the chronic infection has higher risk of developing liver cirrhosis and hepatocellular carcinoma (HCC) (Chang 2007). It has been reported that two million people were infected by $\mathrm{HBV}$ and 650,000 died due to complication of HCC. Development of chronic infection into cirrhosis and HCC was allegedly related to the viral titer, genotype and subgenotype, as well as Hbx protein (Kew 2011; El-Serag 2012; Sunbul 2014). Infection by HBV genotype $C$ has higher risk in developing cirrhosis and HCC, while infection with HBV genotype B may develop HCC without cirrhosis (Chemin and Zoulim 2009; Di Bisceglie 2009).

\footnotetext{
* Current address: Department of Pharmacy, Faculty of Science, Tadulako University, Central Sulawesi, Indonesia

** Deceased

* Corresponding Author

E-mail Address: anita@fa.itb.ac.id
}

Hbx protein is the smallest HBV protein which is composed of 154 amino acids and has four disulphide bonds, between Cys7-Cys69, Cys61-Cys115, Cys78Cys137, and Cys17-Cys143 (Sidhu et al. 2014). Hbx is known for its function in cell cycle modulation, apoptosis regulation and modulation of signal transduction. Hbx influences signal transduction of Ras, Raf, c-jun, MAPK, NFk-B, Jak-Stat, FAK, PKC, Srcdependent signalling and PI3K. Hbx also inactivates p53 by directly interacting with $\mathrm{Hbx}$ C-terminal region, therefore inhibiting p53 localization into nucleus (Kew 2011; Shlomai et al. 2014; Hussein 2016).

In this study, we performed overproduction, purification and refolding of $\mathrm{Hbx}$ protein in Escherichia coli BL21(DE3) for further application in p53 interaction study as well as screening for prospective inhibitors. The gene encoding for $\mathrm{Hbx}$ gene was first optimized according to the codon preference and \%GC of E. coli BL21. Optimation of Hbx overproduction was performed by variations of IPTG concentration as inducer. Investigation of soluble and insoluble $\mathrm{Hbx}$ was performed at three 
different induction temperatures. As insoluble $\mathrm{Hbx}$ was overproduced, purification and refolding steps was performed simultaneously according to previous study (Basu and Leong 2012) with modification. The refolded Hbx protein was characterized using reducing and non-reducing SDS-PAGE and thermal shift assay approach.

\section{Materials and Methods}

\subsection{Bacterial Strains, Plasmids and Culture Media}

E. coli strains, TOP 10 for cloning and BL21(DE3) for protein expression, are maintained at the laboratory of Pharmaceutical Biotechnology, School of Pharmacy, Institut Teknologi Bandung. The plasmid pET16b carrying codon-optimized $\mathrm{Hbx}$ gene was obtained as synthetic gene, purchased from GenScript ${ }^{\circledR}$. The bacteria were grown at $37^{\circ} \mathrm{C}$ in Luria Bertani (LB) medium containing $100 \mu \mathrm{g} / \mathrm{ml}$ ampicillin and the protein expression was induced by isopropyl $\beta$-d-1-thiogalactopyranoside (IPTG).

\subsection{Construction of codon-optimized $\mathbf{H b x}$ Gene}

The sequence of $H b x$ gene was obtained from HBV subgenotype B3 deposited in NCBI database accession number KX429626.1. The codon optimization was performed using OPTIMIZER software (http://genomes.urv.es/OPTIMIZER/) and Codon Optimization Tool (https://sg.idtdna.com/ CodonOpt), based on the codon preference of E. coli B obtained from http://www.kazusa.or.jp/codon/ and \%GC of E. coli, i.e. 51.9\%. For the insertion to expression vector pET16b, NdeI site was introduced at 5' end of the $\mathrm{Hbx}$ gene and BamHI site was introduced at 3' end of $\mathrm{Hbx}$ gene. Codon-optimized $\mathrm{Hbx}$ gene was then obtained as synthetic gene in pET16b. The plasmid was then characterized by migration analysis, restriction analysis using NdeI and BamHI and by nucleotide sequencing.

\subsection{Overproduction, Purification Refolding of $\mathbf{H b x}$}

and

The Hbx protein was overproduced in $E$. coli BL21(DE3) as fusion protein with His-tag. Recombinant E. coli was grown at $37^{\circ} \mathrm{C}, 150 \mathrm{rpm}$ and the expression of Hbx was induced at IPTG concentration of $0.025-0.2 \mathrm{mM}$. The cells were harvested at four hours after addition of IPTG. For quick check of protein expression, one $\mathrm{ml}$ culture was centrifuged at $4,500 \mathrm{~g}$ for 10 minutes and then the cell pellet was lysed using SDS-PAGE sample buffer containing 10\% DTT. The sample was heated at $95^{\circ} \mathrm{C}$ for $10 \mathrm{~min}$ and then subjected to $12 \% \mathrm{SDS}$ PAGE analysis. After determination of optimum IPTG concentration, the expression of soluble or insoluble Hbx was investigated at three different induction temperatures, 17,25 , and $37^{\circ} \mathrm{C}$. After 4 hours induction, cells were collected by centrifugation at 4,500 $\mathrm{g}$ for 10 minutes and then resuspended in LEW buffer ( $50 \mathrm{mM} \mathrm{NaH}_{2} \mathrm{PO}_{4}, 300 \mathrm{mM} \mathrm{NaCl}, \mathrm{pH}$ 8.0). Cell lysis was then performed using sonication at $15 \mathrm{sec}$ pulse on and 15 sec pulse off, with $60 \%$ amplitude for $10 \mathrm{~min}$. The supernatant and cell debris were then mixed with SDS-PAGE sample buffer and heated at $95^{\circ} \mathrm{C}$ for $10 \mathrm{~min}$. Analysis of the soluble and insoluble fraction was performed using $12 \%$ SDS-PAGE.

The inclusion bodies containing $\mathrm{Hbx}$ protein were washed sequentially using Triton X-100 and Tris- $\mathrm{HCl}$ buffer, followed by solubilization in $8 \mathrm{M}$ urea. The solubilized inclusion bodies were then subjected to purification and refolding. Purification of Hbx protein was performed using Ni-NTA column due to presence of His-tag in the Hbx protein. Refolding step was performed prior to elution using $2 \mathrm{M}$ urea, $0.1 \mathrm{mM} \mathrm{GSH}, 0.01 \mathrm{mM}$ GSSG with variation of arginine, 0.1 and $0.25 \mathrm{M}$. First refolding step was performed using $3 \mathrm{ml}$ refolding buffer and second refolding step was performed using $10 \mathrm{ml}$ refolding buffer with incubation at $4^{\circ} \mathrm{C}$ for $18 \mathrm{~h}$. The protein was then eluted using $2 \mathrm{M}$ urea, $0.25 \mathrm{M}$ arginine, 0.1 mM GSH, 0.01 mM GSSG and 100 mM imidazole.

\subsection{Characterization of Refolded $\mathrm{Hbx}$}

The refolded $\mathrm{Hbx}$ protein was then analyzed using reducing and non-reducing 12\% SDS-PAGE. In the non-reducing SDS-PAGE, the protein was mixed with SDS-PAGE sample buffer without the presence of $\beta$-mercaptoethanol. Prior to analysis using thermal shift assay, the sample was subjected to buffer exchange using $2 \mathrm{M}$ urea and $0.25 \mathrm{M}$ arginine. The Hbx protein was then mixed with Sypro Orange and the melting curve profile was obtained using qPCR machine at $20-95^{\circ} \mathrm{C}$. 


\section{Results}

Construction of codon-optimized $\mathrm{Hbx}$ gene was performed based on amino acid sequence from HBV subgenotype B3. Codon optimization was performed according to the \%GC and codon preference of $E$. coli B. The required Codon Adaptation Index (CAI) is $>0.7$, with \% GC close to \%GC E. coli, 51.06\%. The Hbx gene sequence was first optimized using OPTIMIZER and the result showed CAI of 0.641 and \%GC of $62.1 \%$. The sequence was further optimized manually using Codon Optimization Tool and the result showed CAI 0.705 with \%GC of $50.9 \%$, which met the requirements. The codon-optimized $\mathrm{Hbx}$ gene sequence showed $72.04 \%$ similarity to the original sequence (Figure $1 \mathrm{~A})$, while the protein sequence deduced from both sequences showed $100 \%$ similarity (Figure 1B). NdeI site and BamHI site were added to the $5^{\text {' }}$ and $3^{\text {' }}$ end of the sequence, respectively, to allow insertion into pET16b. Characterization of recombinant plasmid was performed using migration analysis ans restriction analysis. Migration analysis showed that pET16b-Hbx has shorter migration as compared to pET16b (Figure 1C). Restriction analysis using NcoI and BamHI showed two fragments with experimental size of 5818 and 541 bp (Figure 1D). The results showed correct characters of recombinant plasmid, which were confirmed using nucleotide sequencing (data not shown).

Optimation of Hbx overproduction was performed upon various concentration of IPTG as inducer, $0.025-0.2 \mathrm{mM}$, and the total protein was analyzed using SDS-PAGE. The result showed presence of thick band at $18.4 \mathrm{kDa}$ which was already induced using the lowest concentration of IPTG, which was not present in non-induced sample (Figure 2A). This size was approximately corresponding to the theoretical size of $\mathrm{Hbx}$, which is $17 \mathrm{kDa}$. IPTG at $0.075 \mathrm{mM}$ was then chosen for further experiments. Investigation of the solubity of the expressed $\mathrm{Hbx}$ was performed by fractionation of cell lysate soluble and insoluble fraction. Cell was grown to the midlog phase and then induced with $0.075 \mathrm{mM}$ IPTG for 4 hours at 17,25 , and $37^{\circ} \mathrm{C}$. Cells were then lysed by sonication and the supernatant and cell debris were subjected to SDS-PAGE. The result showed that the $\mathrm{Hbx}$ protein was located at the cell debris at all growth and induction conditions (Figure 2B). The result conveyed that insoluble $\mathrm{Hbx}$ was produced and required further treatment.

The inclusion bodies containing $\mathrm{Hbx}$ protein were then washed and then solubilized in $8 \mathrm{M}$ urea. The denatured protein was then purified using Ni-NTA column due to the presence of His-tag. Refolding steps was performed simultaneously with purification to generate the disulfide bonds present in Hbx. Initially, refolding step was performed using refolding buffer containing $2 \mathrm{M}$ urea, $0.1 \mathrm{mM} \mathrm{GSH}$, $0.01 \mathrm{mM}$ GSSG, and $0.25 \mathrm{M}$ arginine. According to the previous study by Basu and Leong, which used $2 \mathrm{M}$ urea, $0.1 \mathrm{mM}$ GSH, $0.01 \mathrm{mM}$ GSSG with $0.25 \mathrm{M}$ arginine as refolding buffer. Our results showed that the use of this refolding buffer resulted in elution of most Hbx protein during refolding steps. Therefore, only small amount of $\mathrm{Hbx}$ was present during elution (Figure 3A). Based on this result, the composition of refolding buffer was modified by reducing the concentration of arginine into $0.1 \mathrm{M}$. The result showed that only small amount of Hbx protein was washed out during refolding step, while most were obtained at elution stage with relatively high purity (Figure 3B).

Characterization of the refolded $\mathrm{Hbx}$ protein was performed using SDS-PAGE and thermal shift assay. Firstly, SDS-PAGE analysis was performed at reducing and non-reducing conditions. In nonreducing condition, beta-mercaptoethanol was omitted and the result may give information on the presence of disulfide bond. The result showed that after refolding the refolded Hbx protein had longer migration as compared to the denatured $\mathrm{Hbx}$ protein (Figure 4A). These results conveyed that the disulfide bonds were successfully formed during refolding stage. Secondly, thermal shift assay was employed to characterize the refolded Hbx protein. Prior to refolding, the Hbx protein showed high fluoresence intensity as compared to the refolded Hbx (Figure 4B). This result showed that after refolding, the hydrophobic residues of Hbx protein were not exposed at the protein surface. From the first derivative curve, the melting point of denatured $\mathrm{Hbx}$ was $57.5^{\circ} \mathrm{C}$, while the melting point of refolded $\mathrm{Hbx}$ was $53.8^{\circ} \mathrm{C}$ (Figure $4 \mathrm{C}$ ). 


\section{A}

KX429626.1 synthetic Consensus

KX429626.1 synthetic Consensus

KX429626.1 synthetic Consensus

KX429626.1 synthetic Consensus

KX429626.1 Consensus

B

คอบ82309.1 Consensus

\section{คอบ82309.1 synthetic Consensus}

\begin{tabular}{|c|c|c|c|c|c|c|c|c|c|}
\hline 10 & 20 & 30 & 40 & 50 & 60 & 70 & 80 & 90 & 100 \\
\hline
\end{tabular}

ATGGCTGCTAGGCTGTGCTGCCAACTGGATCCTGCGCGGGACGTCCTTTGTCTACGTCCCGTCGGCGCTGRATCCCGCGGACGACCCCTCCCGGGGCCGC

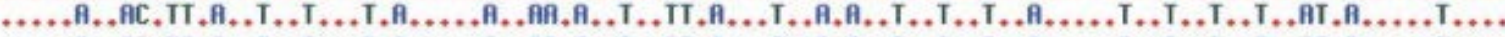

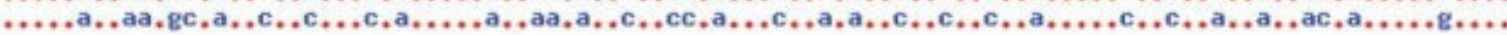

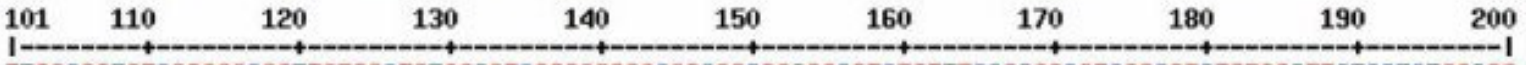

TTGGGGCTCTACCGCCCGCTTCTCCGTCTGCCGTACCGACCGACCACGGGGCGCACCTCTCTTIACGCGGACTCCCCGTCTGTGCCTTCTCATCTGCCGG

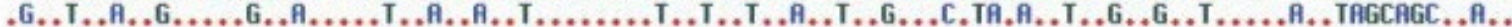

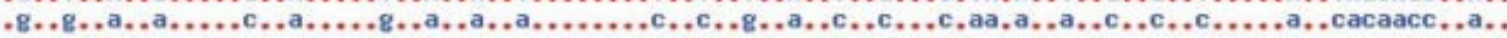

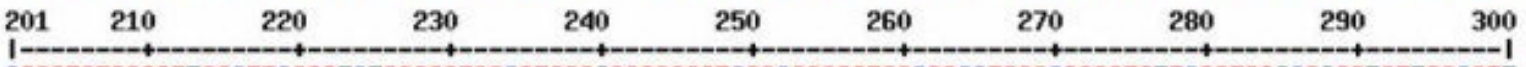

ACCGTGTGCACTTCGCTTCACCTCTGCACGTCGCATGGAGACCACCGTGAACGCCCATCGGAACCTGCCCAAGGTCTTGCATARGAGGACTCTTGGACTT

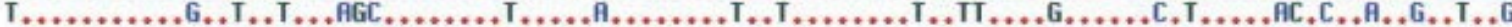

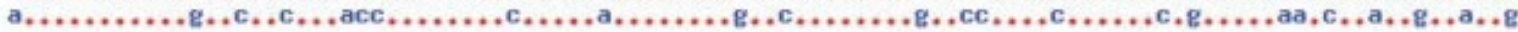

$\begin{array}{llllllllll}301 & 310 & 320 & 330 & 340 & 350 & 360 & 370 & 380 & 390\end{array}$

1----+-

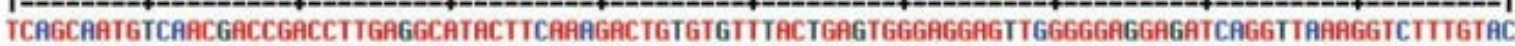

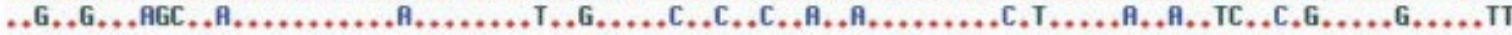

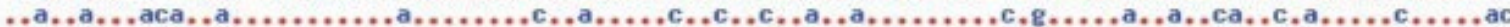

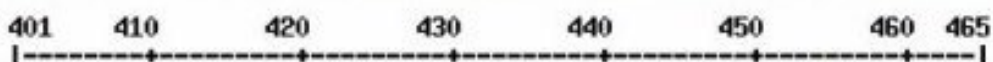

\section{1}

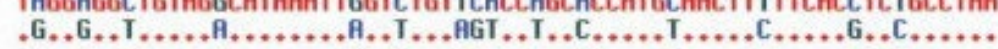

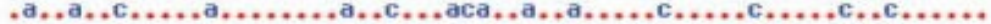

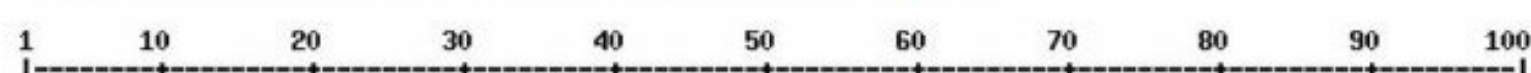

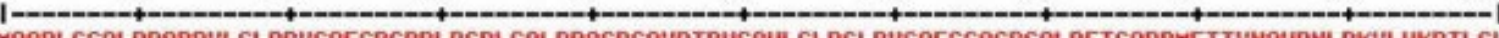

MARRLCCQLDPAROVLCLRPVGAESRGRPLPGPLGALPPASPSAYPTDHGAHLSLRGLPVCAFSSAGPCALRFTSARRMETTVNAHRMLPKVLHKRTLGL

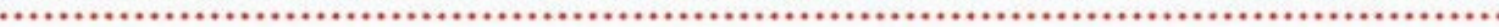

$101 \quad 110 \quad 120 \quad 130 \quad 140 \quad 150154$

SAMSTTDLEAYFKDCVFTEMEEL GEEIRLKVFVLGGCRHKL VCSPAPCNFFTSA

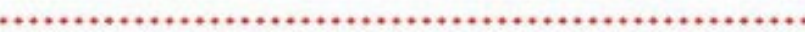

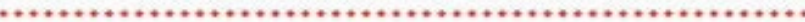

$\mathrm{C}$

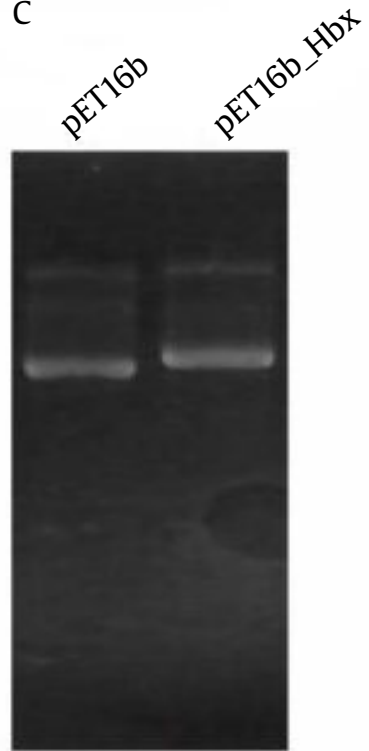

D

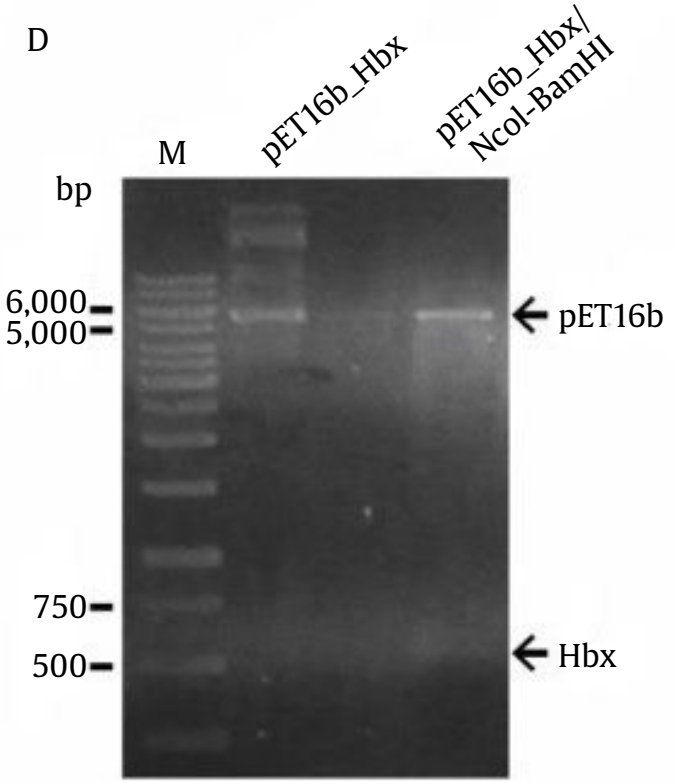

Figure 1. Construction of Hbx synthetic gene and characterization of recombinant plasmid. (A) Alignment of Hbx gene sequences from database and the synthetic construct, (B) alignment of Hbx protein sequences deduced from the database and the synthetic construct, (C) characterization of recombinant pET16b_Hbx using agarose gel electrophoresis, (D) restriction analysis of pET16b_Hbx using NcoI and BamHI. Circular and linear plasmids were analyzed using $1 \%$ agarose gel electrophoresis. M: $1 \mathrm{~kb}$ DNA marker 

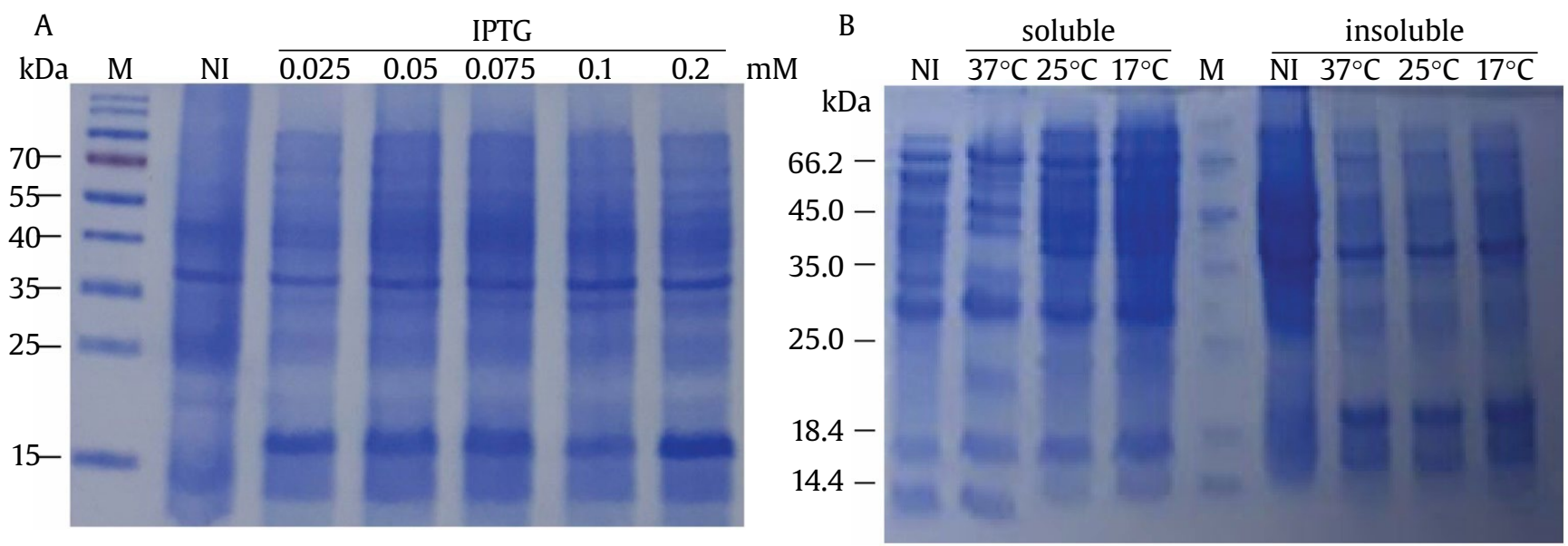

Figure 2. Overproduction of recombinant Hbx in E. coli BL21(DE3). (A) Expression of Hbx upon induction by 0.025-0.2 mM IPTG for 4 hours, (B) expression of Hbx protein in the supernatant of cell lysate and in the cell debris at 17, 25, and $37^{\circ} \mathrm{C}$. Protein analyses were performed using $12 \%$ SDS-PAGE. M: protein marker, NI: non-induced, I: induced

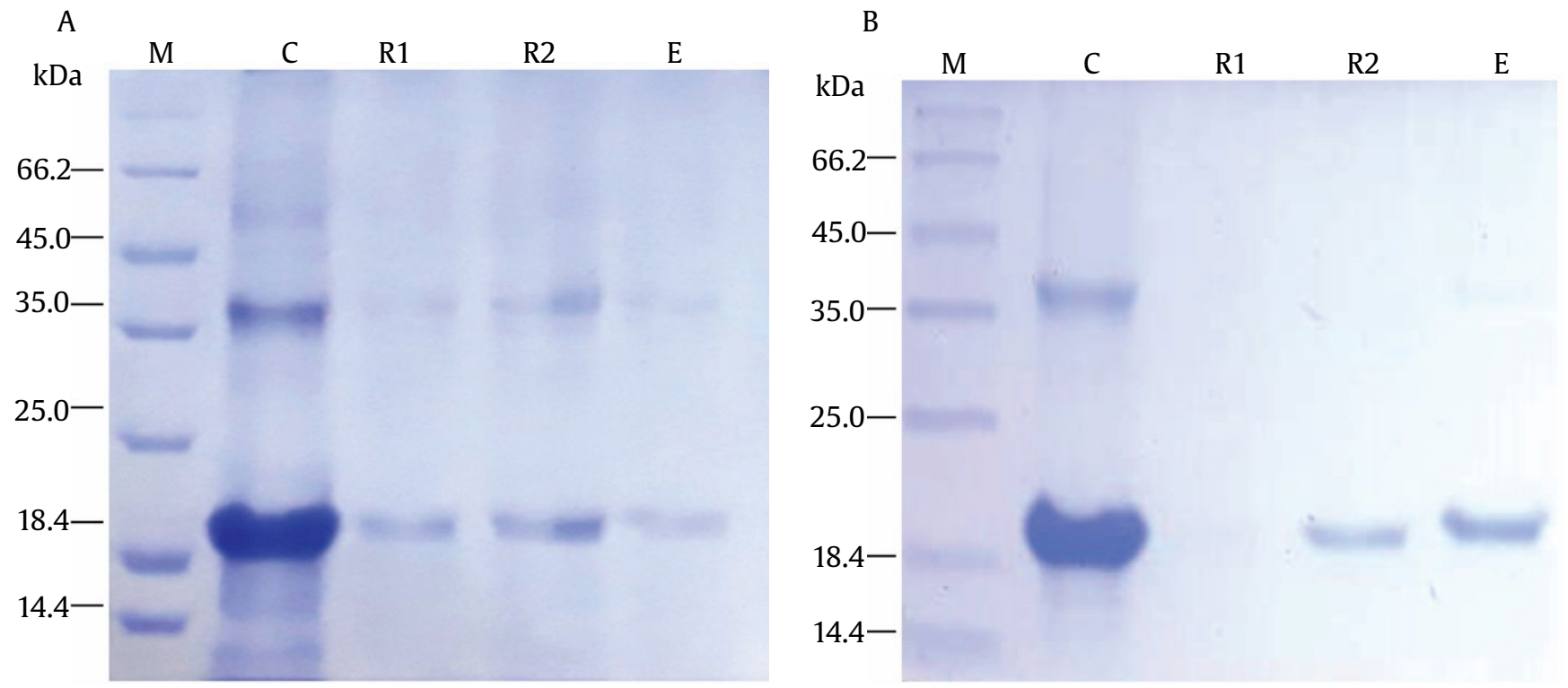

Figure 3. Purification and refolding of Hbx protein from inclusion bodies. The solubilized inclusion bodies was purified using Ni-NTA column and refolded using refolding buffer containing $0.25(\mathrm{~A})$ and $0.1 \mathrm{M}$ arginine. Protein analyses were performed using 12\% SDS-PAGE. M: Protein marker, C: crude, R1: refolding step 1, R2: refolding step 2, E: elution 

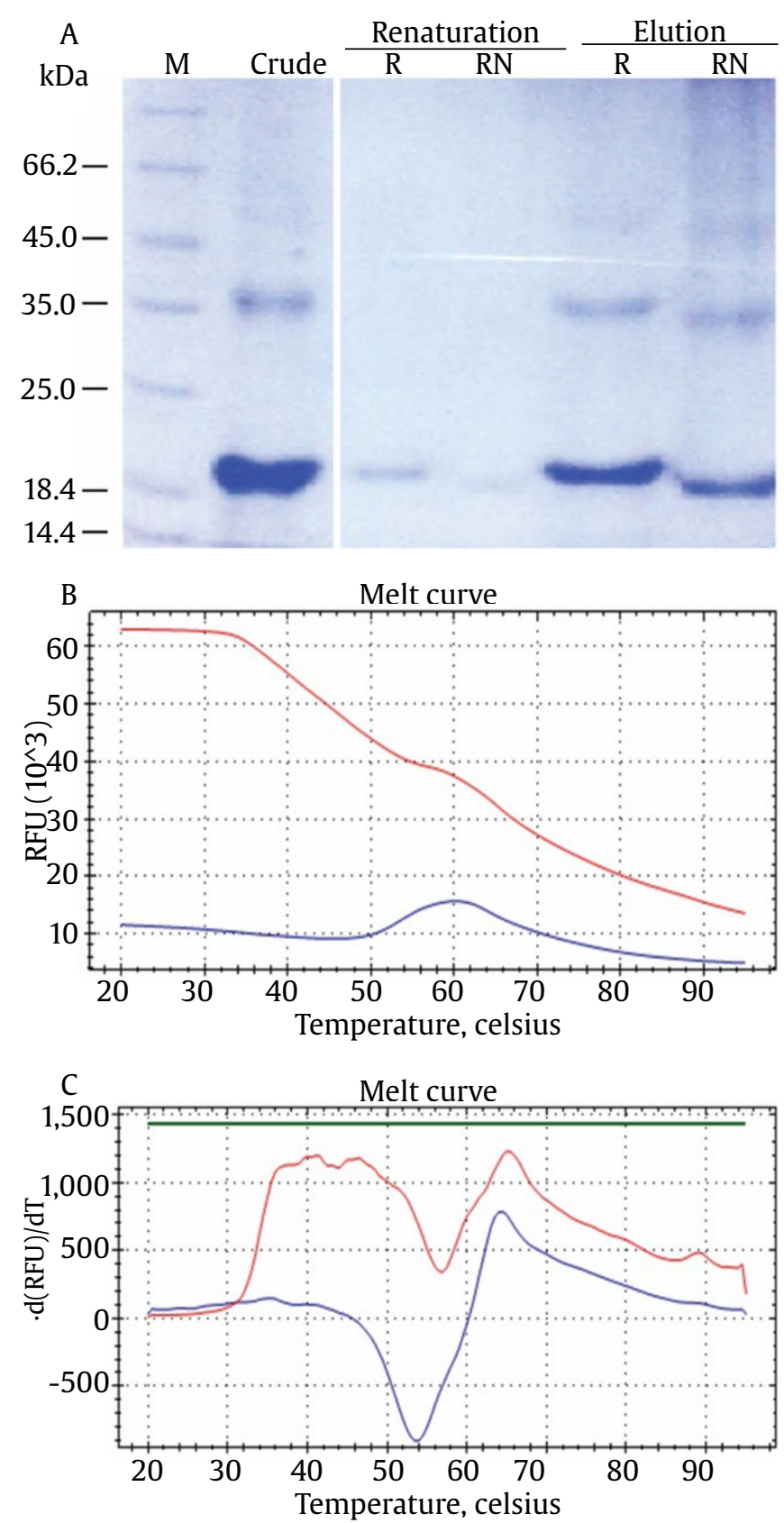

Figure 4. Characterization of refolded Hbx protein using SDS-PAGE and thermal shift assay. The eluted HBx protein was analyzed using reducing and non-reducing SDS-PAGE (A) and thermal shift assay $(B, C)$. Fluorescence intensity of Sypro orange were measured at $20-95^{\circ} \mathrm{C}(\mathrm{B})$ and the first derivative curve was generated to determine protein melt curve (C). M: Protein marker, R: reducing, NR: non-reducing, blue line: before renaturation, red line: after renaturation

\section{Discussion}

The Hbx protein of HBV is one factor thought to be important in development of HCC in chronic hepatitis $B$ infection. Hbx protein is known for its activity in modulating MAPK and JAK1 signal transduction, Wnt/ $\beta$-catenin, NF-k $\beta$, and ERK/JNK transactivation, epigenetic regulation of RAR- $\beta 2$, E-cadherin and p16, as well as modulation of cell cycle regulator protein, such as p21, p15, p16, p27, and Cyclin E (Ali et al. 2014). Hbx protein forms stable secondary structure with alpha-helics, beta-sheet and random coil with four disulfide bonds (Liu et al. 2009; Sidhu et al. 2014). Hbx has BH3-like motif at its carboxy terminus that is responsible for its interaction with $\mathrm{Bcl}-2$ and $\mathrm{Bcl}-\mathrm{XL}$. Interaction of $\mathrm{Hbx}$ with $\mathrm{Bcl}-2$ protein family increases cytosolic calcium concentration, apoptosis and viral DNA replication (Jiang et al. 2016). Hbx also interacts with carboxy terminus of p53, tumor suppressor protein, causing inhibition of p53 entry into nucleus. Therefore, preventing induction of proteins regulated by $\mathrm{p} 53$, such as induction of apoptosis (Wu et al. 2010). To date, three-dimensional structure of $\mathrm{Hbx}$ has not been resolved, only BH3-like motif of Hbx in complex with Bcl-2 has been resolved. This causes difficulty in studying mechanism of interaction with other cellular protein, as well as screening for potential inhibitors.

In order to study the three-dimensional structure of Hbx that will provide essential information about its interaction with other molecules, the overproduction of $\mathrm{Hbx}$ is needed. To date, E. coli is known as the simplest, quickest, and most economical host. Besides, the genome has been fully revealed and a huge number of modification has been performed (Hayat et al. 2018). In this study, not only E. coli was utilized as the host of choice, but codon optimization was conducted as a strategy to increase protein yield. However, production of more complex proteins, E. coli brought about several difficulties, one of which is the issue of protein insolubility, resulting in obtaining protein as inclusion bodies rather than the soluble one. More than half of the amino acids in Hbx protein are hydrophobic. It also has four disulfide bonds in total. Such structure tends to be produced as inclusion bodies in E. coli expression system (Liu et al. 2009). Several studies did produce $\mathrm{Hbx}$ as soluble protein with the help of large protein fusion to improve the folding (Kay et al. 1985; Liu et al. 2009). In this study, different approach was applied; no long fusion protein was added, but a short histidine tag used for protein purification. We were focusing at 
the during-production and downstream processing. Decreasing overproduction temperature to up to $17^{\circ} \mathrm{C}$ was expected to slow down the production, improve folding, and hence increase the yield of soluble protein, but turned out no improve was observed. Despite the abundance of target protein being produced as inclusion bodies, it required additional steps until soluble functional proteins were obtained.

The adaptation of previous study was not suitable for this system as the protein was washed from the column during refolding process done simultaneously with the purification process. Arginine as aggregation inhibitor was known to interact with tryptophan side chains; however, Hbx itself has only one tryptophan amino acid (Tsumoto et al. 2008). Lower concentration of arginine fixed the condition, resulting in soluble $\mathrm{Hbx}$ eluted after the refolding process. Too much arginine in the system could possibly interfere the histidine tag with Nickel column. SDS PAGE and thermal shift assay results showed that disulfide bonds were formed, and more hydrophobic residues were kept inside the tertiary structure of $\mathrm{Hbx}$ as the result of protein solubilization and refolding. Further, the confirmation of proper refolding should be done. There are several methods of choice available, such as Circular Dichroism to analyze the secondary structures, ELISA to confirm antibody anti-HBx recognition, and also in vitro activity assay by observing its ability to trans activate a type of cis-acting regulatory element (Kay et al. 1985; Jameel et al. 1990; Liu et al. 2009). Complete confirmation studies could then be followed by the determination of its three-dimensional structure that will be very beneficial of the study of its interaction with other molecules, especially protein.

\section{Acknowledgements}

Financial support for the project was provided by Indonesian Ministry of Research and Higher Education under PDUPT scheme given to Anita Artarini.

\section{References}

Ali, A., Abdel-Hafiz, H., Suhail, M., Al-Mars, A., Zakaria, M.K., Ahmad, K.F.S., Azhar, E., Chaudhary, A., Qadri, I., 2014. Hepatitis B virus, HBx mutants and their role in hepatocellular carcinoma. World Journal of Gastroenterology. 20, 10238-10248. https://doi. org/10.3748/wjg.v20.i30.10238

Basu, A., Leong, S.S.J., 2012. High productivity chromatography refolding process for Hepatitis B Virus $\mathrm{X}(\mathrm{HBx})$ protein guided by statistical design of experiment studies. Journal of Chromatography A. 1223, 64-71. https:// doi.org/10.1016/j.chroma.2011.12.037
Chang, M.H., 2007. Hepatitis B virus infection. Seminars in Fetal and Neonatal Medicine. 12, 160-167. https://doi. org/10.1016/j.siny.2007.01.013

Chemin, I., Zoulim, F., 2009. Hepatitis B virus induced hepatocellular carcinoma. Cancer letters. 286, 52-59. https://doi.org/10.1016/j.canlet.2008.12.003

Di Bisceglie, A.M., 2009. Hepatitis B and hepatocellular carcinoma. Hepatology. 49, 56-60. https://doi. org/10.1002/hep.22962

El-Serag, H.B., 2012. Epidemiology of viral hepatitis and hepatocellular carcinoma. Gastroenterology.142, 12641273. https://doi.org/10.1053/j.gastro.2011.12.061

Hayat, S.M.G., Farahani, N., Golichenari B., Sahebkar, A., 2018. Recombinant protein expression in Escherichia coli (E. coli): what we need to know. Current Pharmaceutical Design. 24, 718-725. https://doi.org/10.2174/138161 2824666180131121940

Hussein, R.A., 2016. Structural Investigations of Hepatitis $B$ virus $X$ protein. University of Hamburg. Available at: http://ediss.sub.uni-hamburg.de/ volltexte/2016/7716/. [Date accessed: 27 August 2020]

Jameel, S., Siddiqui, A., Maguire, H.F., Rao, K.V., 1990. Hepatitis B virus X protein produced in Escherichia coli is biologically functional. Journal of virology. 64, 3963-3966. https://doi.org/10.1128/jvi.64.8.39633966.1990

Jiang, T., Liu, M., Wu, J., Shi, Y., 2016. Structural and biochemical analysis of Bcl-2 interaction with the hepatitis B virus protein HBx. Proc. Natl. Acad. Sci. USA. 113, 2074-2079. https://doi.org/10.1073/pnas.1525616113

Kay, A., Mandart, E., Trepo, C., Galibert., F., 1985. The HBV HBx gene expression in $E$. coli is recognised by sera from hepatitis patients. The EMBO journal. 4, 1287-1292. https://doi.org/10.1002/j.1460-2075.1985.tb03774.x

Kew, M.C., 2011. Hepatitis B virus x protein in the pathogenesis of hepatitis B virus-induced hepatocellular carcinoma. Journal of gastroenterology and hepatology. $26,144-$ 152. https://doi.org/10.1111/j.1440-1746.2010.06546.x

Liu, D., Zou, L., Li, W., Wang, L., Wu, Y., 2009. High-level expression and large-scale preparation of soluble HBX antigen from Escherichia coli. Biotechnology and applied biochemistry. 54, 141-147. https://doi. org/10.1042/BA20090116

Shlomai, A., de Jong, Y.P., Rice, C.M., 2014. Virus associated malignancies: the role of viral hepatitis in hepatocellular carcinoma. Seminars in Cancer Biology. 26, 78-88. https://doi.org/10.1016/j. semcancer.2014.01.004

Sidhu, K., Kumar, S., Reddy, VS., Kumar, V., 2014. Mass spectrometric determination of disulfide bonds in the biologically active recombinant $\mathrm{HBx}$ protein of hepatitis B virus. Biochemistry. 53, 4685-4695. https://doi.org/10.1021/bi500140t

Sunbul, M., 2014. Hepatitis B virus genotypes: global distribution and clinical importance. World Journal of Gastroenterology. 20, 5427. https://doi.org/10.3748/ wjg.v20.i18.5427

Tsumoto, K., Umetsu M., Kumagai, I., Ejima, D., Philo, J.S., Arakawa, T., 2008. Role of arginine in protein refolding, solubilization, and purification. Biotechnology Progress. 20, 1301-1308. https://doi.org/10.1021/ bp0498793

Wu, Z.J., Zhu, Y., Huang, De-Rong., Wang, Z.Q., 2010. Constructing the HBV-human protein interaction network to understand the relationship between HBV and hepatocellular carcinoma. Journal of experimental and clinical cancer research. 29, 146. https://doi. org/10.1186/1756-9966-29-146 